\title{
Removal of foreign bodies in the upper gastrointestinal tract in adults: European Society of Gastrointestinal Endoscopy (ESGE) Clinical Guideline
}

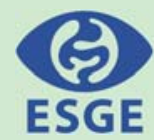

Authors

Institutions
Michael Birk ${ }^{1}$, Peter Bauerfeind ${ }^{2}$, Pierre H. Deprez ${ }^{3}$, Michael Häfner ${ }^{4}$, Dirk Hartmann ${ }^{5}$, Cesare Hassan ${ }^{6}$, Tomas Hucl ${ }^{7}$, Gilles Lesur ${ }^{8}$, Lars Aabakken ${ }^{9}$, Alexander Meining ${ }^{1}$

Institutions are listed at end of article.
Bibliography

DOI http://dx.doi.org/

10.1055/s-0042-100456

Published online: 10.2.2016

Endoscopy 2016; 48: 489-496

(c) Georg Thieme Verlag KG

Stuttgart · New York

ISSN 0013-726X

Corresponding author

Alexander Meining, MD

Department of Internal

Medicine I, University of Ulm

Albert-Einstein-Allee 23

89081 Ulm

Germany

alexander.meining@uniklinikulm.de

Fax: +49-731-44636

This Guideline is an official statement of the European Society of Gastrointestinal Endoscopy (ESGE). It addresses the removal of foreign bodies in the upper gastrointestinal tract in adults.

\section{Recommendations}

Nonendoscopic measures

1 ESGE recommends diagnostic evaluation based on the patient's history and symptoms. ESGE recommends a physical examination focused on the patient's general condition and to assess signs of any complications (strong recommendation, low quality evidence).

2 ESGE does not recommend radiological evaluation for patients with nonbony food bolus impaction without complications. We recommend plain radiography to assess the presence, location, size, configuration, and number of ingested foreign bodies if ingestion of radiopaque objects is suspected or type of object is unknown (strong recommendation, low quality evidence).

3 ESGE recommends computed tomography (CT) scan in all patients with suspected perforation or other complication that may require surgery (strong recommendation, low quality evidence). 4 ESGE does not recommend barium swallow, because of the risk of aspiration and worsening of the endoscopic visualization (strong recommendation, low quality evidence).

5 ESGE recommends clinical observation without the need for endoscopic removal for management of asymptomatic patients with ingestion of blunt and small objects (except batteries and magnets). If feasible, outpatient management is appropriate (strong recommendation, low quality evidence). 6 ESGE recommends close observation in asymptomatic individuals who have concealed packets of drugs by swallowing ("body packing"). We recommend against endoscopic retrieval. We recommend surgical referral in cases of suspected packet rupture, failure of packets to progress, or intestinal obstruction (strong recommendation, low quality evidence).
Endoscopic measures

7 ESGE recommends emergent (preferably within 2 hours, but at the latest within 6 hours) therapeutic esophagogastroduodenoscopy for foreign bodies inducing complete esophageal obstruction, and for sharp-pointed objects or batteries in the esophagus. We recommend urgent (within 24 hours) therapeutic esophagogastroduodenoscopy for other esophageal foreign bodies without complete obstruction (strong recommendation, low quality evidence).

8 ESGE suggests treatment of food bolus impaction in the esophagus by gently pushing the bolus into the stomach. If this procedure is not successful, retrieval should be considered (weak recommendation, low quality evidence).

The effectiveness of medical treatment of esophageal food bolus impaction is debated. It is therefore recommended, that medical treatment should not delay endoscopy (strong recommendation, low quality evidence).

9 In cases of food bolus impaction, ESGE recommends a diagnostic work-up for potential underlying disease, including histological evaluation, in addition to therapeutic endoscopy (strong recommendation, low quality evidence).

10 ESGE recommends urgent (within 24 hours) therapeutic esophagogastroduodenoscopy for foreign bodies in the stomach such as sharp-pointed objects, magnets, batteries and large/long objects. We suggest nonurgent (within 72 hours) therapeutic esophagogastroduodenoscopy for medium-sized blunt foreign bodies in the stomach (strong recommendation, low quality evidence).

11 ESGE recommends the use of a protective device in order to avoid esophagogastric/pharyngeal 
damage and aspiration during endoscopic extraction of sharppointed foreign bodies. Endotracheal intubation should be considered in the case of high risk of aspiration (strong recommendation, low quality evidence).

12 ESGE suggests the use of suitable extraction devices according to the type and location of the ingested foreign body (weak recommendation, low quality evidence).

13 After successful and uncomplicated endoscopic removal of ingested foreign bodies, ESGE suggests that the patient may be discharged. If foreign bodies are not or cannot be removed, a case-by-case approach depending on the size and type of the foreign body is suggested (weak recommendation, low quality evidence).

\section{Abbreviations \\ CT computed tomography \\ ESGE European Society of Gastrointestinal Endoscopy \\ GRADE Grading of Recommendations Assessment, Development and Evaluation \\ NASPGHAN North American Society for Pediatric Gastroente- rology, Hepatology and Nutrition}

\section{Introduction}

$\nabla$

Foreign body ingestion and food bolus impaction are encountered commonly in clinical practice. The majority of foreign body ingestions occur in the pediatric population. Most ingested foreign bodies ( $80 \%-90 \%)$ pass spontaneously. However, approximately $10 \%-20 \%$ of cases of foreign body ingestion require endoscopic removal, while less than $1 \%$ will need surgery for foreign body extraction or to treat complications [1-7]. This Guideline aims to provide endoscopists with a comprehensive review of diagnostic and therapeutic treatment options for swallowed foreign bodies in adults. For the management of ingested foreign bodies in children we refer to the clinical report of the NASPGHAN Endoscopy Committee [8].

\section{Methods \\ $\nabla$}

The European Society of Gastrointestinal Endoscopy (ESGE) commissioned this Guideline and appointed a guideline leader (A.M.) who invited the listed authors to participate in the project development. The guideline development process included meetings and online discussions among members of the guideline committee during January 2015 and July 2015. Key questions were prepared by the coordinating team (A. M. and M. B.). A systematic literature search in PubMed/MEDLINE and the Cochrane Library was conducted using the search terms "foreign body AND ingestion AND gastrointestinal NOT child" and "foreign bodies AND endoscopy AND gastrointestinal NOT child" to identify publications since 2000 on this topic, to prepare evidence-based statements on the key questions. Articles were first selected by title, their relevance was then assessed by reviewing full-text articles, and publications with content that was considered irrelevant were excluded. All selected articles were graded by the level of evidence and strength of recommendation according to the Grading of Recommendations Assessment, Development and
Table 1 Classification of swallowed foreign bodies.

\begin{tabular}{|l|l|}
\hline Type & Examples \\
\hline Blunt objects & $\begin{array}{l}\text { Round objects: coin, button, toy } \\
\text { Batteries, magnets }\end{array}$ \\
\hline $\begin{array}{l}\text { Sharp-pointed } \\
\text { objects }\end{array}$ & $\begin{array}{l}\text { Fine objects: needle, toothpick, bone, safety-pin, } \\
\text { glass pieces } \\
\text { Sharp irregular objects: partial denture, razor blade }\end{array}$ \\
\hline Long objects & $\begin{array}{l}\text { Soft objects: string, cord } \\
\text { Hard objects: toothbrush, cutlery, screwdriver, pen, } \\
\text { pencil }\end{array}$ \\
\hline Food bolus & With or without bones \\
\hline Others & Packets of illegal drugs \\
\hline
\end{tabular}

Evaluation (GRADE) system. Evidence tables were generated for each key question, summarizing the level of evidence of the available studies. Draft proposals were presented to the entire group for general discussion and voting during a plenary meeting held in July 2015 (Munich, Germany).

In August 2015, a draft prepared by M.B. and A.M. was sent to all group members. After agreement on a final version, the manuscript was submitted to the journal Endoscopy for publication. The journal subjected the manuscript to peer review, and the manuscript was amended to take into account the reviewers' comments. The final revised manuscript was agreed upon by all the authors.

\section{Recommendations and statements $\nabla$}

\section{Foreign body ingestion and food bolus impaction}

Ingestion of true foreign bodies (i.e. nonfood objects) occurs more frequently in children than in adults. In adults, esophageal food bolus impaction is a much more common problem with an estimated annual incidence of $13 / 100000$ people [9]. True foreign body ingestion in adults, either intentional or unintentional, appears more often in the elderly population; in patients with psychiatric disorders developmental delay, or alcohol intoxication; and in prisoners seeking secondary gain [1 - 7]. A classification of foreign bodies is listed in Table 1; some examples are shown in Fig.1.

\section{Nonendoscopic measures}

\section{ESGE recommends diagnostic evaluation based on the patient's history and symptoms. ESGE recommends a physical examination focused on the patient's general condition and to assess signs of any complications (strong recommendation, low quality evidence).}

For communicative adults, history of ingestion including timing, type of ingested foreign body and onset of symptoms is usually reliable. In mentally impaired adults and in cases of intentional foreign body ingestion for secondary gain (e.g. by prisoners), a medical evaluation can be difficult. Patients with esophageal foreign bodies, particularly impacted food boluses, are almost always symptomatic and can specify the onset of symptoms and localize discomfort exactly. However, the area of discomfort often does not correlate with the site of impaction $[1,2,4,10]$. Esophageal foreign bodies result in symptoms such as dysphagia, odynophagia, or retrosternal pain; sore throat, foreign body sensation, retching, and vomiting are also very common. Respiratory symptoms include choking, stridor, or dyspnea and can result from as- 


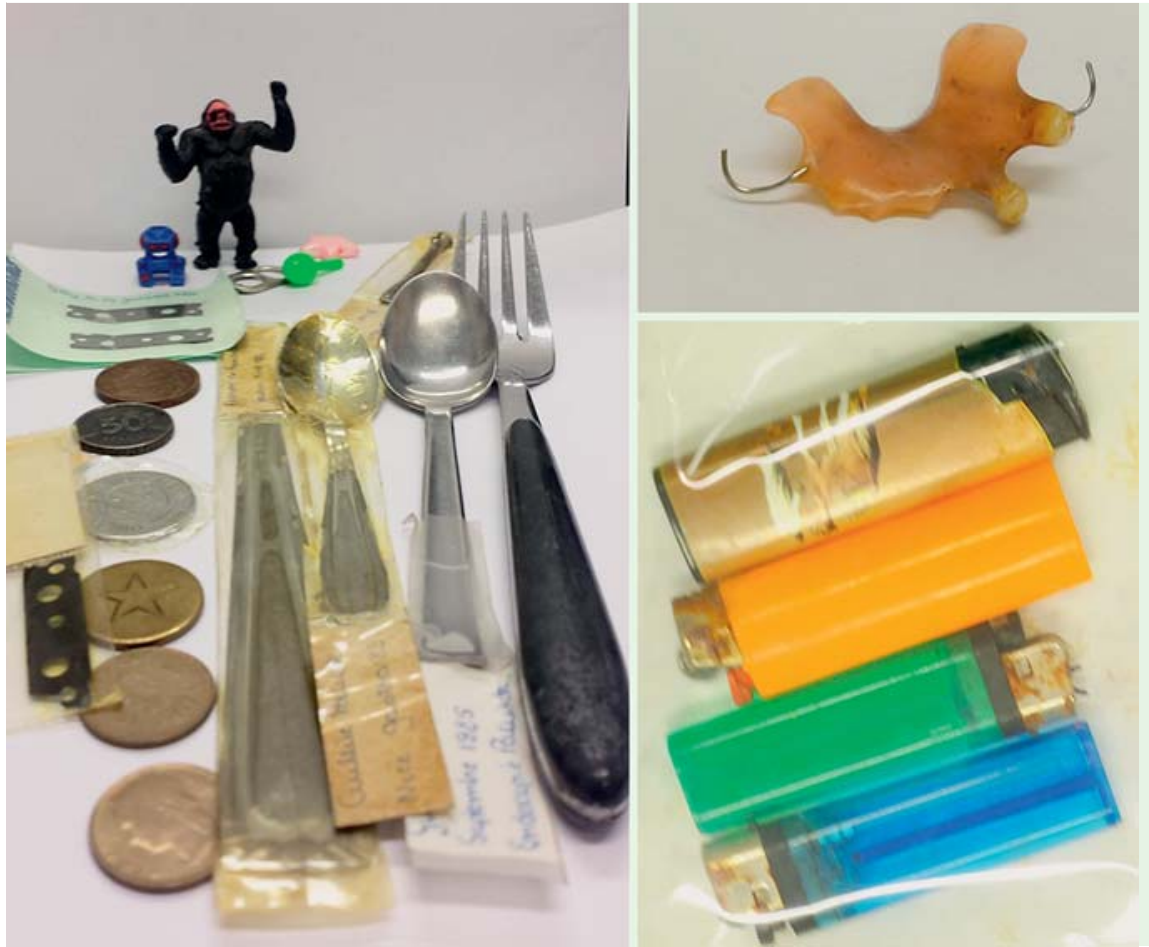

Fig. 1 Examples of foreign bodies retrieved from the upper gastrointestinal tract (courtesy of Dr. Patrick Druez). piration of saliva or from tracheal compression by the foreign body. Hypersalivation and inability to swallow any liquids are suspicious for complete esophageal obstruction [3-7,10-15]. When the foreign body has passed the esophagus, the majority of patients remain asymptomatic but a sensation of foreign body, with dysphagia, can persist for several hours and thus can mimic a persisting foreign body impaction.

Physical examination is mandatory to detect ingestion-related complications such as small-bowel obstruction. Symptoms indicating perforation include fever, tachycardia, peritonitis, subcutaneous crepitus, and swelling of the neck or chest. Lung examination should be performed to assess the presence of wheezing or aspiration [2-7].

\section{ESGE does not recommend radiological evaluation for patients with nonbony food bolus impaction without complications. We recommend plain radiography to assess the presence, location, size, configuration, and number of ingested foreign bodies if ingestion of radiopaque objects is suspected or type of object is unknown (strong recommendation, low quality evidence).}

When a history of foreign body ingestion is elicited, a radiographic evaluation of the neck, chest and abdomen is recommended to assess the presence, location, size, configuration, and number of ingested objects. Furthermore, complications such as aspiration, free mediastinal/peritoneal air, or subcutaneous emphysema can be detected $[1-7,10-14,16-20]$. To minimize exposure to radiation, plain radiography is recommended as the initial screening method, but false-negative rates are as high as $47 \%$ [5]. Therefore, biplanar radiography is recommended if the object is not detected on plain radiographs.

Most true foreign bodies can be identified radiographically; however, thin metal objects, wood, plastic, and glass, and fish or chicken bones are not readily seen ( $\bullet$ Table 2 ). For radiographic evaluation of food bolus impaction, false-negative rates of about $87 \%$ have been reported, so X-ray is not sufficient and not requir-
Table 2 Classification of foreign bodies according to their radiodensity.

\begin{tabular}{|c|c|}
\hline Radiodensity & Foreign body \\
\hline $\begin{array}{l}\text { Can mostly be identified on } \\
\text { radiography }\end{array}$ & $\begin{array}{l}\text { True foreign bodies (i. e. nonfood objects) } \\
\text { Steak bones }\end{array}$ \\
\hline $\begin{array}{l}\text { Cannot (regularly) be iden- } \\
\text { tified on radiography }\end{array}$ & $\begin{array}{l}\text { Food bolus } \\
\text { Fish or chicken bones } \\
\text { Wood } \\
\text { Plastic } \\
\text { Glass } \\
\text { Thin metal objects }\end{array}$ \\
\hline
\end{tabular}

ed in patients with nonbony food bolus impaction and without clinical signs of perforation $[1-7,10,11,13,14,16-18]$.

ESGE recommends computed tomography (CT) scan in all patients with suspected perforation or other complication that may require surgery (strong recommendation, low quality evidence).

In adults, fish bones and other bone fragments are the most commonly ingested foreign bodies and are likely to become lodged in the upper esophagus with a high risk of perforation $[1-3,6,7,11$, $14,15,21]$. However, radiography does not always reliably detect radiolucent foreign bodies, especially fish bones. Even when fish bones are sufficiently radiopaque to be visualized on radiographs, large soft-tissue masses and fluid can obscure the minimal calcium content of the bone, particularly in obese patients [22]. Results of a prospective study with 358 patients who had swallowed fish bones revealed that radiography had a sensitivity of only $32 \%$ [23]. In these cases, CT scan, with a sensitivity from $90 \%$ to $100 \%$ and a specificity of $93.7 \%$ to $100 \%$, is significantly superior to radiography $[6,17,18,22,24]$.

If perforation is suspected based on the clinical or radiological findings, CT is indicated. With CT, the shape, size, location, and depth of the impacted foreign body and the surrounding tissue can be visualized, which is important in determining treatment 
options and evaluating the risks of endoscopic management. CT not only provides better anatomic information, but can also detect other complications such as abscess formation, mediastinitis, or aortic/tracheal fistulas [13,16-19,24-26].

Another difficulty is that the presence of free gas under the diaphragm is almost never seen in foreign body perforation of the gastrointestinal tract. Because the perforation is caused by impaction and progressive erosion of the foreign body through the intestinal wall, the site of perforation becomes covered by fibrin, omentum, or adjacent loops of bowel. This limits the passage of large amounts of intraluminal air into the peritoneal cavity. Free intraperitoneal air is therefore a poor radiologic sign. The region of perforation can be identified on CT scan as a thickened intestinal segment, localized pneumoperitoneum, regional fatty infiltration, or associated intestinal obstruction [26].

Besides for perforation, there are indications for surgical treatment in foreign body ingestion in cases of complications that cannot be resolved endoscopically (e.g. bleeding) or after unsuccessful attempts at endoscopic recovery. Impaction of the foreign body out of endoscopic reach and small-bowel obstruction also require surgery.

ESGE does not recommend barium swallow because of the risk of aspiration and worsening of the endoscopic visualization (strong recommendation, low quality evidence).

In the setting of radiological diagnostic evaluation, use of a barium swallow is not recommended because of the risk of aspiration and because coating of the foreign body and esophageal mucosa with contrast interferes with endoscopic visualization [ $1-5$, $18,19]$. If an esophageal foreign object is assumed to be present but cannot be detected on plain radiography, oral administration of a water-soluble radiocontrast medium (e.g. Gastrografin; Bracco Diagnostics Inc.) can be considered, except when esophageal obstruction is suspected clinically. In the latter cases, watersoluble agents are contraindicated because they are hypertonic and can cause pulmonary edema if aspirated [7]. Generally, radiocontrast evaluation should not delay any necessary endoscopic procedure.

ESGE recommends clinical observation without the need for endoscopic removal for management of asymptomatic patients with ingestion of blunt and small objects (except batteries and magnets). If feasible, outpatient management is appropriate (strong recommendation, low quality evidence).

The majority of ingested foreign bodies ( $80 \%-90 \%$ ) pass through the gastrointestinal tract spontaneously and without complications $[1-3,6,7,27]$. Impaction, perforation, or obstruction often occur at areas of physiological narrowing or angulations. Areas of physiological narrowing include the upper esophageal sphincter, aortic arch, left main stem bronchus, lower esophageal sphincter, pylorus, ileocecal valve, and anus; the duodenal sweep is a physiological angulation. Once foreign bodies have traversed the esophagus, most objects pass within 4-6 days, or in rare cases in up to 4 weeks. Generally, objects greater than $2-2.5 \mathrm{~cm}$ in diameter will not pass through the pylorus or ileocecal valve and objects longer than $5-6 \mathrm{~cm}$ will not pass through the duodenal sweep [1,2,7,20,27].

Conservative outpatient management by means of clinical observation is appropriate for asymptomatic patients with blunt objects in the stomach that are smaller than $2-2.5 \mathrm{~cm}$ in diameter and $5-6 \mathrm{~cm}$ in length. Patients should be instructed to be aware of signs of perforation or small-bowel obstruction and to observe their stools continuously. In the absence of symptoms, weekly radiographs are sufficient to document the progression of the foreign body. If the foreign body fails to pass beyond the stomach within $3-4$ weeks, it should be extracted endoscopically $[1-3,6$, $7,27]$.

ESGE recommends close observation in asymptomatic individuals who have concealed packets of drugs by swallowing ("body packing"). We recommend against endoscopic retrieval. We recommend surgical referral in cases of suspected packet rupture, failure of packets to progress, or intestinal obstruction (strong recommendation, low quality evidence).

The term "body packing" refers to smuggling of drugs by concealment in the gastrointestinal tract. Illegal drugs (most often cocaine or heroin) are packed within latex condoms or balloons and are swallowed or inserted into the rectum in several parcels. The packets can usually be seen radiographically. Rupture and leakage of the contents can lead to fatal intoxication. Therefore, endoscopic removal should not be attempted and a conservative approach is recommended instead, comprising inpatient treatment, clinical observation, whole bowel irrigation, and radiographic follow-up for observing passage of the parcels. Since the failure rate of the conservative approach is only $2 \%-5 \%$, it is always advisable in asymptomatic individuals with "body packed" drugs. Symptomatic individuals present signs of either intoxication or bowel obstruction and will require surgery. In cases of impaction of the packets in the bowel, surgical referral is also indicated $[1-3,6,7]$.

\section{Endoscopic measures}

ESGE recommends emergent (preferably within 2 hours, but at latest within 6 hours) therapeutic esophagogastroduodenoscopy for foreign bodies inducing complete esophageal obstruction, and for sharp-pointed objects and batteries in the esophagus. We recommend urgent (within 24 hours) therapeutic esophagogastroduodenoscopy for other esophageal foreign bodies without complete obstruction (strong recommendation, low quality evidence).

Esophageal foreign objects and food bolus impacted in the esophagus should be removed within 24 hours because delay decreases the likelihood of successful removal and increases the risk of complications $[1-7,21]$. The risk for major complications (i.e., perforation with or without mediastinitis, retropharyngeal abscess and aortoesophageal fistula) increases 14.1 times with foreign bodies impacted for more than 24 hours in the esophagus [28].

Patients with clinical signs of complete esophageal obstruction (i. e., hypersalivation and inability to swallow liquids) have a high risk for aspiration and require an emergent (preferably within 2 hours, but at the latest within 6 hours) endoscopic intervention. The rate of perforation caused by ingested sharp-pointed objects is up to $35 \%$, therefore it is recommended that these foreign bodies should be extracted from the esophagus in an emergent setting also $[1-7,13,21,25,28,29]$. Button batteries or small disk batteries (as used in watches, hearing aids, calculators, and other small electronic devices) can very quickly cause damage in the esophagus, resulting in perforation or fistula due to pressure necrosis, electrical discharge, or chemical injury. The narrow lumen of the esophagus allows mucosal contact with both poles of the battery with subsequent electrical burns. Furthermore, necrosis can result from leakage of the alkaline substances contained in the battery. Batteries also contain heavy me- 
tals, but in small amounts that are unlikely to result in toxicity. Ingestion of cylindrical batteries is rare ( $0.6 \%$ of ingestions) and cause symptoms less frequently $[1-3,5-7,13]$.

ESGE suggests treatment of food bolus impaction in the esophagus by gently pushing the bolus into the stomach. If this procedure is not successful retrieval should be considered (weak recommendation, low quality evidence).

The effectiveness of medical treatment of esophageal food bolus impaction is debated. It is therefore recommended, that medical treatment should not delay endoscopy (strong recommendation, low quality evidence).

The primary method to treat food bolus impaction is the push technique, with success rates of over $90 \%$ and minimal complications. Before the food bolus is pushed into the stomach, an attempt to bypass the bolus with the endoscope should be made to assess any obstructive esophageal pathology beyond the impacted food. Even if this is not possible, most food boluses can be safely pushed into the stomach by using air insufflation and gentle pushing pressure. Placing the endoscope on the right side of the bolus may allow an easier and safer passage into the stomach because the gastroesophageal junction angulates to the left of the patient. Larger boluses can be broken apart with an endoscopic accessory before pushing the smaller pieces into the stomach safely.

If significant resistance is encountered, pushing should not be continued because of the high incidence of underlying esophageal pathology. Applying excessive force in these cases will lead to an increased risk of perforation. Impacted food boluses that cannot be pushed into the stomach, especially those containing bones or sharp edges, must be treated with en bloc retrieval or piecemeal removal after fragmentation, using different types of grasping forceps, polypectomy snares, retrieval net, or Dormia basket $[1-7,10,14,29,30,31]$.

Medical treatment of esophageal food bolus impaction with glucagon has been investigated in several studies [1-4,32-34]. Whether a similar effect can be achieved by using butylscopolamine has not been studied and remains questionable. The utility of glucagon in easing the passage of the bolus into the stomach has been reported to be variable and there seems to be less success in the setting of a fixed anatomic obstruction. Esophageal food bolus impaction is frequently associated with an esophageal anatomic abnormality (e.g. strictures): thus medical treatment will not be very effective in most cases and should therefore not delay endoscopic removal. Although the success rate with glucagon is low, some authors recommend its use as an initial therapy for esophageal food bolus impaction because of its safety and limited side-effect profile. However, the use of glucagon is commonly associated with nausea and vomiting and could potentially increase the risk of perforation and aspiration in the presence of a severe impaction.

\footnotetext{
In cases of food bolus impaction, ESGE recommends a diagnostic work-up for potential underlying disease, including histological evaluation, in addition to therapeutic endoscopy (strong recommendation, low quality evidence).
}

An underlying esophageal pathology is found in more than $75 \%$ of patients presenting with food bolus impaction $[1-7,10,12$, $14,15,19,25]$. The most frequently associated abnormalities are esophageal (mainly peptic) strictures (more than 50\%) and eosinophilic esophagitis (about 40\%). Less frequently, esophageal cancer or esophageal motility disorders, such as achalasia, diffuse esophageal spasm, and nutcracker esophagus, are causes of food bolus impaction. Lack of appropriate follow-up for patients has been shown to be a predictor for recurrent food impactions [5]. Therefore, in all patients a diagnostic work-up after extraction of foreign bodies is recommended to detect any underlying disease $[14,29]$.

Typical endoscopic features in individuals suffering from eosinophilic esophagitis are longitudinal and vertical furrows, trachealization of the esophagus, esophageal edema, and mucosal fragility ("crêpe paper esophagus"). The presence of white exudates is an additional typical finding and these are thought to be clusters of eosinophils. Fixed rings and strictures are complications of eosinophilic esophagitis and potentially result in permanent narrowing of the esophagus. However, in about $10 \%$ of patients the findings are either very subtle or the esophagus appears normal. Biopsies of the upper, middle, and lower thirds of the esophagus, and from those areas where visible white exudates suggest nests of eosinophils, should be obtained at the initial procedure or in a repeat endoscopy to evaluate for eosinophilic esophagitis [30, 35-37]. Esophageal strictures or Schatzki rings can be safely and effectively dilated concurrently in the absence of substantial mucosal damage. Often there is mucosal abrasion or erythema as a result of the food having been lodged in the esophagus for an extended period. In this case, the patient should be prescribed proton pump inhibitor therapy with dilation being performed $2-4$ weeks later [3-7].

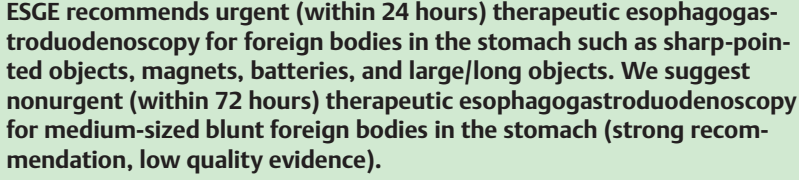
troduodenoscopy for foreign bodies in the stomach such as sharp-pointed objects, magnets, batteries, and large/long objects. We suggest nonurgent (within 72 hours) therapeutic esophagogastroduodenoscopy for medium-sized blunt foreign bodies in the stomach (strong recommendation, low quality evidence).

Although the majority of sharp-pointed objects in the stomach will pass without incident, the risk of complications is as high as $35 \%$. Therefore, it is recommended to retrieve a sharp-pointed object in the stomach or proximal duodenum endoscopically if this can be accomplished safely, considering the patient's fasting status and risk of aspiration $[2,6,7]$.

Because of the attracting forces between ingested magnets or between a single magnet and metallic foreign bodies swallowed at the same time, ingestion of magnets can cause pressure necrosis, fistula, perforation, occlusion, or volvulus. Urgent (within 24 hours) endoscopic removal is recommended even if only one magnet is evident on radiographs or the patient's history suggests ingestion of only one magnet. Additional, undetected magnets or other ingested metal objects together with a magnet can lead to severe gastrointestinal injury $[1-3,5]$.

Some authors suggest endoscopic retrieval of batteries beyond the esophagus emergently, others only when there are coexisting signs of gastrointestinal injury [1-3,5-7]. Most button and small disk batteries in the stomach will pass the gastrointestinal tract without any complications. The risk of electrical burns resulting from ingested batteries in the stomach is low compared with the risk from batteries lodged in the esophagus. Taking into account the danger of liquefaction necrosis due to battery leakage, removal of batteries from the stomach within 24 hours seems to be appropriate. Once the duodenum has been passed, $85 \%$ pass through the remaining intestine within 72 hours.

Medium-sized foreign bodies with a diameter wider than 2$2.5 \mathrm{~cm}$ will normally not pass the pylorus and should retrieved. 
Table 3 Timing of endoscopic intervention in foreign body ingestions: emergent is preferably within 2 hours, but at latest within 6 hours; urgent, within 24 hours; nonurgent, within 72 hours.

\begin{tabular}{|lll|}
\hline Object type & Location & Timing \\
\hline Battery & Esophagus & Emergent \\
\hline Magnet & Stomach/small bowel & Urgent \\
\hline & Esophagus & Urgent \\
\hline Sharp-pointed foreign body & Stomach/small bowel & Urgent \\
\hline Blunt and small foreign body $<2-2.5 \mathrm{~cm}$ diameter & Esophagus & Emergent \\
\hline Blunt and medium-sized foreign body $>2-2.5 \mathrm{~cm}$ diameter & Stomach/small bowel & Usopht \\
\hline & Esophagus & Urgent \\
\hline Large foreign body $>5-6 \mathrm{~cm}$ & Stomach/small bowel & Nonurgent \\
\hline & Esophagus & Urgent \\
\hline Food bolus & Stomach/small bowel & Nonurgent \\
\hline
\end{tabular}

Objects longer than 5-6cm usually become lodged in the duodenal curve and must be removed as an urgent procedure because of a risk of perforation in $15 \%-35 \%$ of cases. An overview on timing of endoscopy according to the type of ingested foreign body and its location in the gastrointestinal tract is given in $\square$ Table 3 $[1-3,5-7]$.

ESGE recommends the use of a protective device in order to avoid esophagogastric/pharyngeal damage and aspiration during endoscopic extraction of sharp-pointed foreign bodies. Endotracheal intubation should be considered in the case of high risk of aspiration (strong recommendation, low quality evidence).

Airway protection is of special concern during foreign body removal and food bolus extraction. Standard-sized overtubes that extend past the upper esophageal sphincter not only protect the airways but also facilitate passage of the endoscope during removal of multiple objects or during piecemeal extraction of an impacted food bolus. Endotracheal intubation may be needed if the patient is not cooperative or if there is a high risk of aspiration (i.e., full stomach, proximal esophageal location of the foreign body, food bolus impaction).

Overtubes also help to protect the esophageal/pharyngeal mucosa from lacerations during retrieval of sharp objects ( Fig.2). Longer overtubes of $45-60 \mathrm{~cm}$ that extend past the lower esophageal sphincter should be used during removal of sharp-pointed objects distal to the esophagus. Use of a transparent cap or latex rubber hood ( $\mathbf{F i g . 3}$ ) is recommended to prevent mucosal injury from sharp-pointed objects if there is no overtube available. There is one randomized controlled trial demonstrating that transparent cap-assisted endoscopy is a safe and effective method in the management of foreign bodies in the upper esophagus, with a significantly shorter operation time and clearer visual field compared with conventional esophagogastroduodenoscopy $[2-7,12,29,38]$.

ESGE suggests the use of suitable extraction devices according to the type and location of the ingested foreign body (weak recommendation, low quality evidence).

Flexible endoscopy is the best diagnostic and therapeutic approach in the management of foreign bodies and food bolus impaction in the upper gastrointestinal tract, with success rates greater than $95 \%$ and complication rates of $0 \%-5 \%[2,3,5,6,11$,

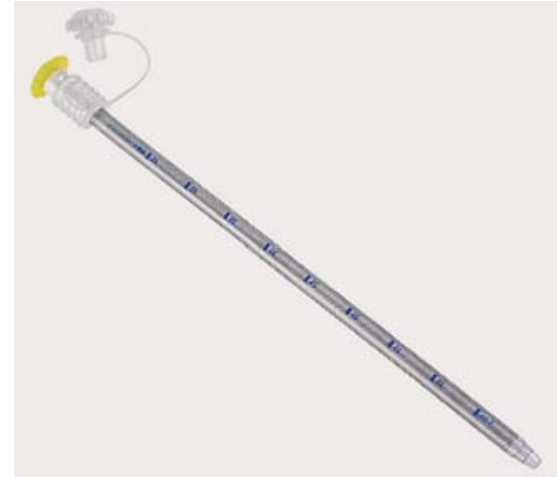

Fig. 2 Overtube used in endoscopic retrieval of ingested foreign bodies (Guardus overtube - permission granted by US Endoscopy).

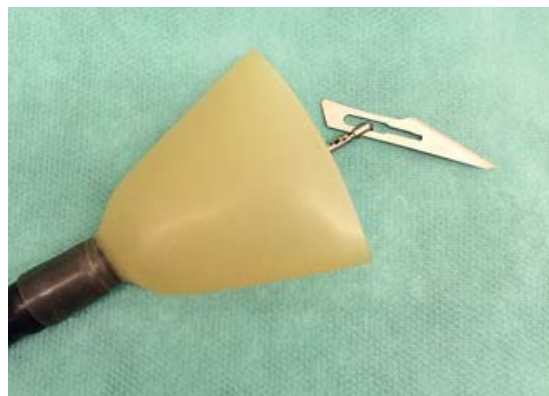

Fig. 3 Latex rubber hood used to prevent mucosal injury during retrieval of a scalpel blade.

$12,14,19,25,29,39]$. The choice of retrieval device is determined by the size and shape of the foreign body ( $\odot$ Table 4; $\odot$ Fig. 4 and - Fig.5), by the endoscope length and instrument channel, and by the endoscopist's preference and practice. Removal of foreign bodies with standard biopsy forceps is rarely successful because of the forceps' small opening width, and cannot therefore be recommended [3]. Retrieval forceps have a large variety of jaw configurations: rat-tooth, alligator-tooth, or shark-tooth. Retrieval graspers with two to five prongs can be useful for retrieving soft objects, but not for harder or heavy objects because the grip is not secure enough. Polypectomy snares are widely available and inexpensive. Endoscopic baskets may be useful for round objects, and retrieval nets or bags can provide a more secure grasp for some foreign bodies (coins, batteries, magnets) and for en bloc removal of food boluses.

With sharp objects, the foreign body should be grasped in such a position that the sharp or pointed end trails distally to the endoscope, thus lowering the risk of a procedure-related perforation or mucosal damage during extraction. Long foreign bodies must 


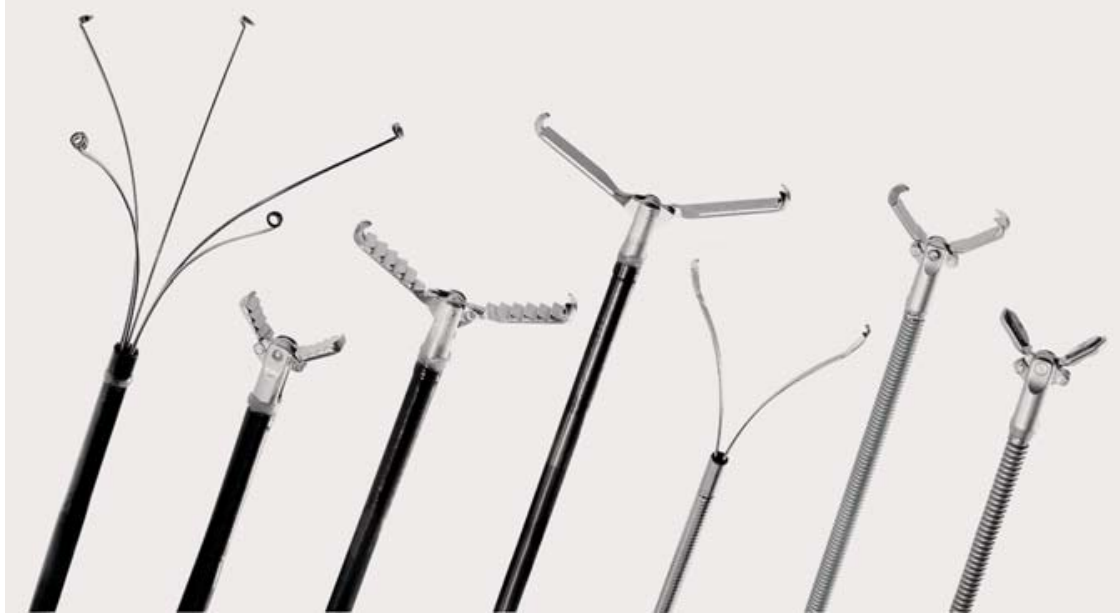

Fig. 4 Retrieval graspers and grasping forceps (image provided by Olympus Europe, Hamburg, Germany).

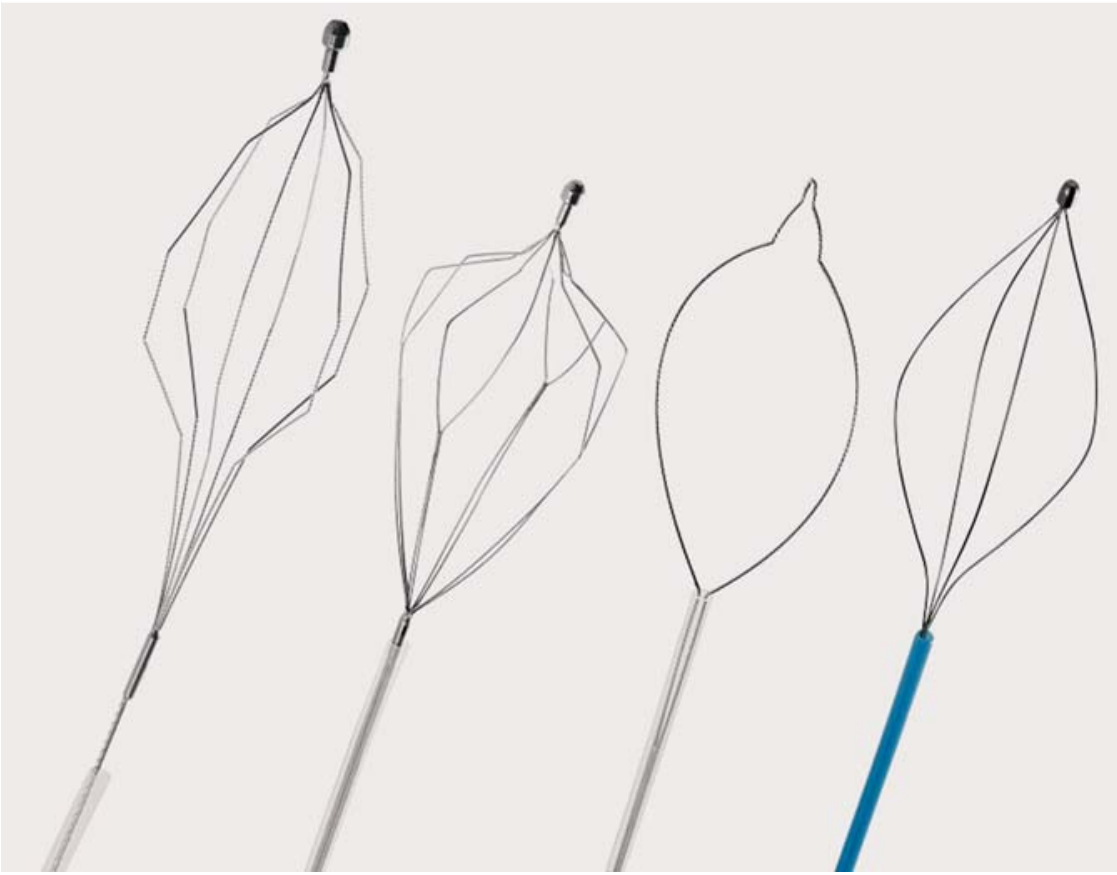

Fig. 5 Baskets and snare (image provided by Olympus Europe, Hamburg, Germany).

Table 4 Overview of retrieval devices.

\begin{tabular}{|c|c|}
\hline Object type & Appropriate retrieval devices \\
\hline Blunt objects & $\begin{array}{l}\text { Grasping forceps, retrieval graspers, polypectomy snare, } \\
\text { basket, retrieval net }\end{array}$ \\
\hline $\begin{array}{l}\text { Sharp-poin- } \\
\text { ted objects }\end{array}$ & $\begin{array}{l}\text { Grasping forceps, polypectomy snare, basket, retrieval } \\
\text { net } \\
\text { Transparent cap, latex rubber hood }\end{array}$ \\
\hline Long objects & Polypectomy snare, basket \\
\hline Food bolus & $\begin{array}{l}\text { Grasping forceps, retrieval graspers, polypectomy snare, } \\
\text { basket, retrieval net }\end{array}$ \\
\hline
\end{tabular}

be grabbed at the very end of the object to allow retrograde removal through the esophagus. Grasping the object near the center would turn the object so that its length was radially across the lumen, preventing it from being pulled through the sphincters and the esophagus.

Before endoscopy, it is useful to practice grasping an object similar in shape to the ingested foreign body, using different accessories to determine the most appropriate available retrieval device [2-7].
After successful and uncomplicated endoscopic removal of ingested foreign bodies, ESGE suggests that the patient may be discharged. If foreign bodies are not or cannot be removed, a case-by-case approach depending on the size and type of the foreign body is suggested (weak recommendation, low quality evidence).

Most patients with foreign body ingestion or food bolus impaction can be treated as outpatients after endoscopic therapy. Consideration should be given to admitting patients for observation after technically difficult extraction, when there has been ingestion of multiple objects or foreign bodies associated with a high risk for complications (i.e., sharp-pointed objects, batteries, magnets, objects larger than $5-6 \mathrm{~cm})$, and when there is extensive mucosal injury due to the foreign body ingestion or endoscopic treatment.

If the foreign body cannot be retrieved endoscopically, inpatient treatment and close clinical observation is mandatory for sharppointed objects and batteries. Radiographic follow-up examinations should be performed to assess the object's passage through the gastrointestinal tract. Daily radiographs are recommended for sharp-pointed objects. For batteries beyond the duodenum, plain radiography every 3-4 days is adequate. Surgery must be considered for removal of dangerous foreign bodies that have 
passed the ligament of Treitz and fail to progress within 3 days after ingestion. Long objects lodged in the duodenum need surgical therapy when endoscopic efforts fail [1 -3,5-7,27].

ESGE Guidelines represent a consensus of best practice based on the available evidence at the time of preparation. They might not apply in all situations and should be interpreted in the light of specific clinical situations and resource availability. Further controlled clinical studies may be needed to clarify aspects of these statements, and revision may be necessary as new data appear. Clinical considerations may justify a course of action at variance with these recommendations. ESGE Guidelines are intended to be an educational device for providing information that may assist endoscopists in providing care to patients. They are not rules and should not be construed as establishing a legal standard of care or as encouraging, advocating, requiring, or discouraging any particular treatment.

\section{Competing interests: None}

\section{Institutions}

${ }^{1}$ Department of Gastroenterology, Universitätsklinikum Ulm, Ulm, Germany Department of Internal Medicine, Division of Gastroenterology, University Hospital Zurich, Zurich, Switzerland

Department of Hepatogastroenterology, Cliniques Universitaires Saint-Luc, Université Catholique de Louvain, Brussels, Belgium

${ }^{4}$ Department of Internal Medicine, St. Elisabeth Hospital,Vienna, Austria

Department of Gastroenterology, Sana Klinikum Lichtenberg, Berlin, Germany

${ }^{6}$ Department of Gastroenterology, Nuovo Regina Margherita Hospital, Rome, Italy

Department of Gastroenterology and Hepatology, Institute for Clinical and Experimental Medicine, Prague, Czech Republic

${ }^{8}$ Department of Gastroenterology, Ambroise Paré Hospital, Boulogne, France

${ }^{9}$ Department of Medical Gastroenterology, Rikshospitalet University Hospital, Oslo, Norway

\section{References}

1 Ambe $P$, Weber SA, Schauer $M$ et al. Swallowed foreign bodies in adults. Dtsch Arztebl Int 2012; 109: 869-875

2 ASGE Standards of Practice Committee. Ikenberry SO, Kue TL, Andersen $M A$ et al. Management of ingested foreign bodies and food impactions. Gastrointest Endosc 2011; 73: 1085-1091

3 Dray X, Cattan P. Foreign bodies and caustic lesions. Best Pract Res Clin Gastroenterol 2013; 27: 679-689

$4 \mathrm{Ko} \mathrm{HH,} \mathrm{Enns} \mathrm{R.} \mathrm{Review} \mathrm{of} \mathrm{food} \mathrm{bolus} \mathrm{management.} \mathrm{Can} \mathrm{J} \mathrm{Gastroenterol}$ 2008; 22: $805-808$

5 Pfau PR. Removal and management of esophageal foreign bodies. Tech Gastrointest Endosc 2014; 16: 32 - 39

6 Sugawa C, Ono J, Taleb $M$ et al. Endoscopic management of foreign bodies in the upper gastrointestinal tract: A review. World J Gastrointest Endosc 2014; 6: 475 - 481

7 Telford JJ. Management of ingested foreign bodies. Can J Gastroenterol 2005; 19: 599-601

8 Kramer RE, Lerner DG, Lin T et al. Management of ingested foreign bodies in children: a clinical report of the NASPGHAN Endoscopy Committee. J Pediatr Gastroenterol Nutr 2015; 60: 562 - 574

9 Longstreth GF, Longstreth KJ, Yao JF. Esophageal food impaction: epidemiology and therapy. A retrospective, observational study. Gastrointest Endosc 2001; 53: 193-198

10 Ciriza C, García L, Suárez P et al. What predictive parameters best indicate the need for emergent gastrointestinal endoscopy after foreign body ingestion? J Clin Gastroenterol 2000; 31: 23-28

11 Chiu YH, Hou SK, Chen SC et al. Diagnosis and endoscopic management of upper gastrointestinal foreign bodies. Am J Med Sci 2012; 343: $192-195$

12 Conway WC, Sugawa C, Ono $H$ et al. Upper GI foreign body: an adult urban emergency hospital experience. Surg Endosc 2007; 21: 455-460

13 Erbil B, Karaca MA, Aslaner MA et al. Emergency admissions due to swallowed foreign bodies in adults. World J Gastroenterol 2013; 19: 6447-6452
$14 \mathrm{Wu} W T$, Chiu CT, Kuo CJ et al. Endoscopic management of suspected esophageal foreign body in adults. Dis Esophagus 2011; 24: 131-137

15 Zhang S, Cui Y, Gong X et al. Endoscopic management of foreign bodies in the upper gastrointestinal tract in South China: a retrospective study of 561 cases. Dig Dis Sci 2010; 55: 1305-1312

16 Lee JH, Kim HC, Yang DM et al. What is the role of plain radiography in patients with foreign bodies in the gastrointestinal tract? Clin Imaging 2012; 36: $447-454$

17 Liew CJ, Poh AC, Tan TY. Finding nemo: imaging findings, pitfalls, and complications of ingested fish bones in the alimentary canal. Emerg Radiol 2013; 20: 311 - 322

18 Marco De Lucas E, Sádaba P, Lastra García-Barón Pet al. Value of helical computed tomography in the management of upper esophageal foreign bodies. Acta Radiol 2004; 45: 369 - 374

19 Mosca S, Manes $G$, Martino $R$ et al. Endoscopic management of foreign bodies in the upper gastrointestinal tract: report on a series of 414 adult patients. Endoscopy 2001; 33: $692-696$

20 Palta $R$, Sahota A, Bemarki A et al. Foreign-body ingestion: characteristics and outcomes in a lower socioeconomic population with predominantly intentional ingestion. Gastrointest Endosc 2009; 69: 426-433

21 Sung SH, Jeon SW, Son HS et al. Factors predictive of risk for complications in patients with oesophageal foreign bodies. Dig Liver Dis 2011; 43: $632-635$

22 Goh BK, Tan YM, Lin SE et al. CT in the preoperative diagnosis of fish bone perforation of the gastrointestinal tract. AJR Am J Roentgenol 2006; $187: 710-714$

$23 \mathrm{Ngan} J \mathrm{H}$, Fok PJ, Lai EC et al. A prospective study on fish bone ingestion: experience of 358 patients. Ann Surg 1989; 211: 459-462

24 Young CA, Menias CO, Bhalla S et al. CT features of esophageal emergencies. Radiographics 2008; 28: $1541-1553$

25 Chen T, Wu HF, Shi Q et al. Endoscopic management of impacted esophageal foreign bodies. Dis Esophagus 2013; 26: 799-806

26 Goh BK, Chow PK, Quah HM et al. Perforation of the gastrointestinal tract secondary to ingestion of foreign bodies. World J Surg 2006; 30: $372-377$

27 Bisharat $M$, O'Donnell ME, Gibson $N$ et al. Foreign body ingestion in prisoners - the Belfast experience. Ulster Med J 2008; 77: 110-114

28 Loh KS, Tan LK, Smith JD et al. Complications of foreign bodies in the esophagus. Otolaryngol Head Neck Surg 2000; 123: 613-616

$29 \mathrm{Li}$ ZS, Sun ZX, Zou DW et al. Endoscopic management of foreign bodies in the upper-GI tract: experience with 1088 cases in China. Gastrointest Endosc 2006; 64: 485 - 492

30 Kerlin P, Jones D, Remedios $M$ et al. Prevalence of eosinophilic esophagitis in adults with food bolus obstruction of the esophagus. J Clin Gastroenterol 2007; 41: $356-361$

31 Vicari JJ, Johanson JF, Frakes JT. Outcomes of acute esophageal food impaction: success of the push technique. Gastrointest Endosc 2001; 53: $178-181$

32 Al-Haddad M, Ward EM, Scolapio JS et al. Glucagon for the relief of esophageal food impaction does it really work? Dig Dis Sci 2006; 51: $1930-1933$

33 Leopard D, Fishpool S, Winter S. The management of oesophageal soft food bolus obstruction: a systematic review. Ann R Coll Surg Engl 2011; $93: 441-444$

34 Sodeman TC, Harewood GC, Baron TH. Assessment of the predictors of response to glucagon in the setting of acute esophageal food bolus impaction. Dysphagia 2004; 19: 18-21

35 Dellon ES, Gonsalves N, Hirano I et al. ACG clinical guideline: Evidenced based approach to the diagnosis and management of esophageal eosinophilia and eosinophilic esophagitis (EoE). Am J Gastroenterol 2013; 108: $679-692$

36 Waidmann O, Finkelmeier F, Welker MW et al. Endoscopic findings in patients with eosinophilic esophagitis. Z Gastroenterol 2015; 53: $379-384$

37 Enns $R$, Kazemi P, Chung $W$ et al. Eosinophilic esophagitis: Clinical features, endoscopic findings and response to treatment. Can J Gastroenterol 2010; 24: 547-551

38 Zhang S, Wang J, Wang J et al. Transparent cap-assisted endoscopic management of foreign bodies in the upper esophagus: a randomized, controlled trial. J Gastroenterol Hepatol 2013; 28: 1339 - 1342

39 Emara M, Darwiesh EM, Refaey MM et al. Endoscopic removal of foreign bodies from the upper gastrointestinal tract: 5-year experience. Clin Exp Gastroenterol 2014; 7: 249-253 